\title{
Brain Imaging of Human Sexual Response: Recent Developments and Future Directions
}

\author{
Gerben B. Ruesink ${ }^{1}$ • Janniko R. Georgiadis ${ }^{1}$
}

Published online: 23 October 2017

(C) The Author(s) 2017, corrected publication November/2017. This article is an open access publication

\begin{abstract}
Purpose of Review The purpose of this study is to provide a comprehensive summary of the latest developments in the experimental brain study of human sexuality, focusing on brain connectivity during the sexual response.

Recent Findings Stable patterns of brain activation have been established for different phases of the sexual response, especially with regard to the wanting phase, and changes in these patterns can be linked to sexual response variations, including sexual dysfunctions. From this solid basis, connectivity studies of the human sexual response have begun to add a deeper understanding of the brain network function and structure involved.

Summary The study of "sexual" brain connectivity is still very young. Yet, by approaching the brain as a connected organ, the essence of brain function is captured much more accurately, increasing the likelihood of finding useful biomarkers and targets for intervention in sexual dysfunction.
\end{abstract}

Keywords Sexual behavior · MRI · Connectivity · Wanting · Liking $\cdot$ Inhibition

This article is part of the Topical Collection on Female Sexual Dysfunction and Disorders

Janniko R. Georgiadis

j.r.georgiadis@umcg.nl

1 Department of Neuroscience (Section Anatomy), University Medical Center Groningen, University of Groningen, Antonius Deusinglaan 1, Box 196, 9700 AD Groningen, The Netherlands

\section{Introduction}

Recent years have seen spectacular developments in the field of human brain imaging (neuroimaging) that allow researchers to analyze human brain structure and function in greater detail than was ever possible. These neuroimaging approaches have begun to be applied to the study of human sexual behavior as well. Given the prevalence of idiopathic sexual dysfunctions, this development is positive, but for sex researchers or sexologists not trained to deal with brain data, it can be difficult to get a grip on the wealth of often complex results. In this review, we provide a comprehensive summary of the latest developments in the experimental brain study of human sexuality, with a focus on the sexual response. We will argue that brain connectivity approaches hold the highest promise to provoke breakthroughs regarding the mechanisms that govern functional and dysfunctional human sexual responding.

\section{From Activity to Connectivity}

"Neuroimaging" applies to the use of various techniques to visualize the structure and function of the nervous system. This review almost exclusively deals with results obtained by magnetic resonance imaging (MRI). Structural MRI provides information about the size, shape, and integrity of gray (clusters of cell bodies, e.g., in the cortex) and white (bundles of axons) matter. Analytic methods such as voxel-based morphometry (VBM) can provide reliable estimates of local gray and/or white matter volume differences, either within or between subjects. Diffusion tensor imaging (DTI) is an important structural MRI protocol that can reconstruct a threedimensional structural map of the white matter tracts (the structural connections) in the brain. Quantitative meta- 
analyses can combine many data sets to make more reliable inferences about morphological brain features in large populations. An example of this is a study on 1400 human brains from four different datasets that could not substantiate the idea of a clear sexual dimorphism in the human brain [1•].

Functional MRI enables the detection of neural activity over time, typically related to a task, group, physiological or psychological parameter, or individual trait, resulting in functional localization (activation). Again, quantitative metaanalysis methods such as activity likelihood estimation can combine data of multiple activation studies and distill the most robust patterns of activation - those that are likely to resemble functional networks $[2,3 \cdot \bullet]$.

Analysis of functional interaction and communication within the brain is termed "functional connectivity" and is essentially calculated as correlations between neural activities of distinct areas. Functional connectivity can be measured for task-based fMRI data, but also for so-called resting state data. The latter does not require intrusive tasks or paradigms that might keep potentially interesting subject groups (e.g., adolescents) from being studied with regard to their sexual brain function. There are different methods that can analyze functional connectivity; some are model-based, such as psychophysiological interaction analysis (PPI) analysis, which can evaluate a more or less specific connection under different task conditions and/or between groups, whereas others like independent component analysis require no task performance and typically can evaluate larger networks or more networks simultaneously $[4,5]$. Brain networks that are consistently found in functional connectivity studies, either in the resting state or during task execution, include the default mode network, visual network, sensory/motor network, and taskpositive network $[6 \bullet \cdot$. As an example, a study using resting state study found that women had stronger functional connectivity in parts of the default mode network than men did and that the menstrual cycle did not modulate this connectivity. It was concluded that transient activating effects of gonadal hormones could not account for the sexual dimorphism in functional connectivity [7]. Granger causality analysis and dynamic causal models can also provide information about the direction of communication between brain areas [8]. This directed communication between brain areas is called "effective" connectivity.

The most recent analytic developments in neuroimaging aim to capture whole-brain functionality by using tools from the field of network science [9*0]. The premise is that the central nervous system behaves as a network, or a system, that tries to achieve an optimal balance between local specialization and global integration. If a network has both properties, it is said to have a small-world organization, and unless there is a severe neurological condition, this usually applies to human brains $[10,11]$. However, within a small-world organization, the balance might be shifted towards local specialization or global integration. Graph analysis methods can provide a detailed analysis of this small-world organization, for instance by investigating the number and location of network hubs (areas that function to integrate network activity). At least in theory, graph analysis is capable of providing the most profound insights into neural mechanisms contributing to human sexuality.

\section{Modeling Sex}

The term "sexual response" refers to the set of behaviors and functions directly related to sexual stimulation and the pursuit of a sexual goal [12]. Models of the human sexual response aim to provide a template to study and compare a variety of sexual responses, relatively independent of other sexuality characteristics. An example of this is the human sexual pleasure cycle [13, 14•]. This model (Fig. 1)—which underlines the significance of external stimulation next to that of the internal "drive" state (incentive motivation theory) [15, 16] - distinguishes the phases wanting sex, liking sex (or having sex), and inhibiting sex. Sexual orientation, sexual preference, and gender identity are then seen as elements determining what kind of stimuli trigger the sexual pleasure cycle. Clinically, this fits with a distinction between sexual dysfunction (i.e., a problem with the sexual response, e.g., erectile dysfunction) and paraphilia (i.e., an atypical sexual preference, e.g., pedophilia). The use of a model like this facilitates comparison between neuroimaging studies that try to model different elements of the sexual response, while allowing different (neuroscientific) explanations and mechanisms for sexual responsiveness.

\section{Overview of Recent Neuroimaging Studies on Human Sexuality}

We reviewed relevant human neuroimaging studies that were published in the period 2012-2017, distinguishing studies representing the sexual response itself and factors involved in triggering a response (sexual orientation, preference, or gender identity). Regarding the sexual response category, we distinguished studies representing wanting, liking, and inhibition phases. Studies were further categorized according to their methodology, i.e., whether they employed analytic approaches focusing on separate activated brain areas, or more sophisticated methods analyzing brain connectivity and networks (see previous section). This rough categorization showed that in the domain of the sexual response, about twice as many neuroimaging studies were conducted than in other domains of human sexuality, but also that the relative contribution of connectivity studies was greater in the latter. Furthermore, within the sexual response domain, it is obvious 
Fig. 1 The human sexual pleasure cycle. Brain areas relevant to this review are depicted per phase (red: increased brain activity; blue: decreased brain activity). Inhibition can be physiological (pink shading) or deliberate (brown shading). Abbreviations: ACC, anterior cingulate cortex; Amy, amygdala; dIPFC, dorsolateral prefrontal cortex; HT, hypothalamus, OFC, orbitofrontal cortex; SPL, superior parietal lobule; vmPFC, ventromedial prefrontal cortex; VS, ventral striatum (Figure uses information from $[3 \cdot \bullet, 13])$

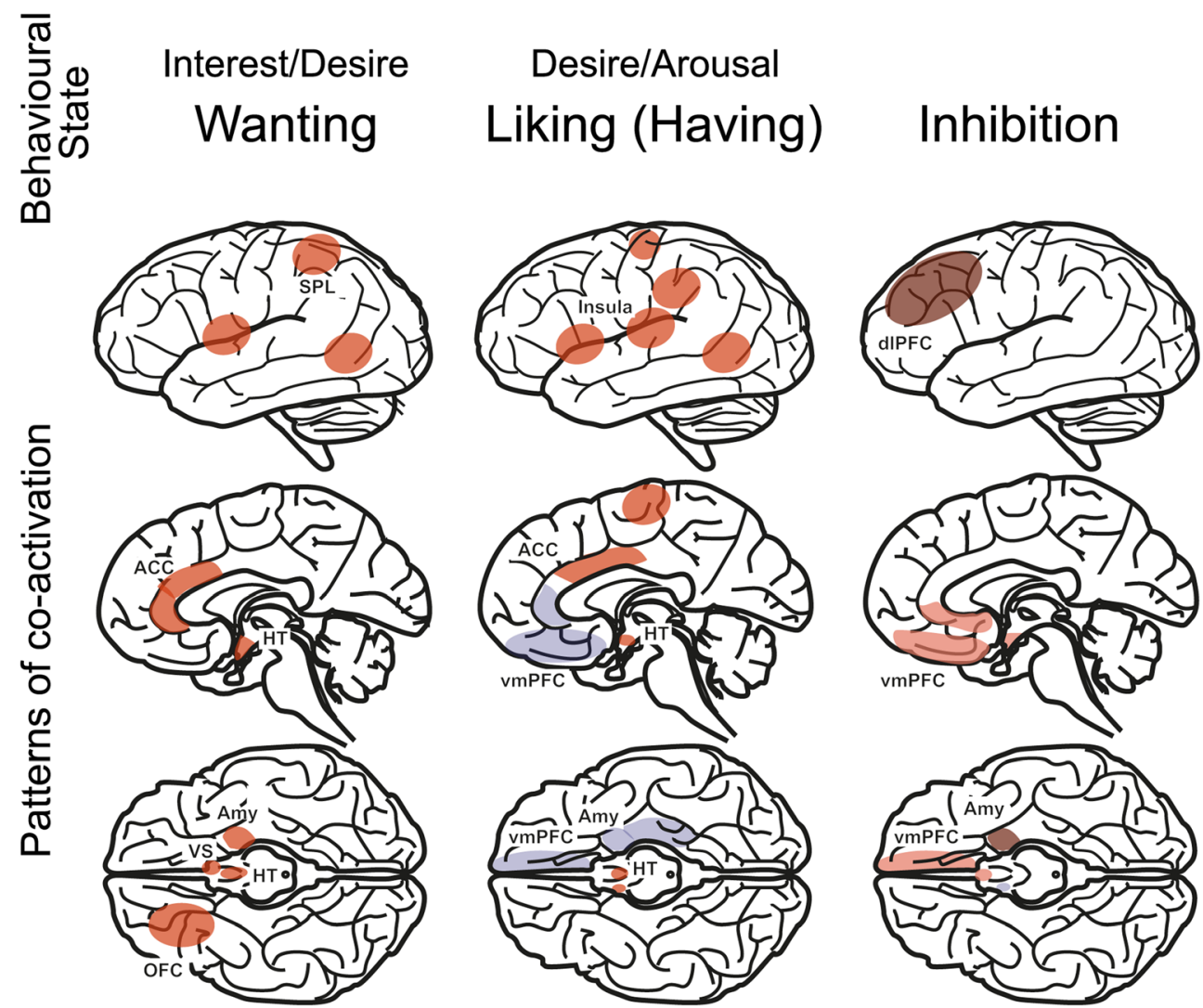

that most of current research efforts are concentrated on the wanting phase, but that connectivity approaches are relatively more common in experiments on the liking phase of the sexual response (Fig. 2).

\section{Current Status of Human Sexual Response Neuroimaging}

Systematic reviews of experimental brain imaging studies of the human sexual response reveal phasedependent patterns of brain activity (Fig. 1) $[3 \cdot \bullet, 13$, 14•, 17]. In their review, Georgiadis and Kringelbach describe a "sexual wanting pattern" including the occipitotemporal cortex, superior parietal lobule, ventral striatum (VS), amygdala/hippocampus, orbitofrontal cortex (OFC), anterior cingulate cortex (ACC), and anterior insula, and a "sexual liking pattern," including the hypothalamus, anterior and posterior insula, ventral premotor cortex, middle cingulate cortex, and inferior parietal lobule [14•]. Using different terms for basically the same distinction, very similar patterns were identified by Poeppl and colleagues performing a quantitative meta-analysis on psycho- and physiosexual elements of the sexual response [3••]. By and large, a sexual response involves very similar brain activation patterns across sexual preferences and gender groups, as long as preferred sexual stimuli are used $[18,19]$. This pattern was refined by a recent meta-analysis, showing a largely consistent pattern across gender groups with statistically significant gender differences mainly in subcortical areas [20]. In addition, there is some indication that phase-dependency in brain response patterns over the course of the sexual response is less marked in women than it is in men [21]. Nevertheless, the stability of the visually evoked sexual wanting pattern was confirmed by scanning subjects on two occasions separated by $1-1.5$ years and showing that the brain response was very similar over time [22]. Furthermore, sexual wanting and liking brain response patterns reflect (parts of) known functional brain networks [6••]. Thus, we conclude that these patterns are robust and should be able to provide a solid basis from which sexual responserelated brain connectivity can be studied.

More than before, experimental designs are being developed that can avoid confounds caused by participant reaction manipulation. Some studies use subliminal (i.e., below the threshold of consciousness) presentations of sexual stimuli, eliminating elaborate cognitive processing [23]. A novel approach involves adding cognitive loading (mental rotation task) to a visual sexual stimulation design to decrease the likelihood of cognitive reaction manipulation [24]. Such 
Fig. 2 Overview of neuroimaging studies on the sexual response from the period of 2012 to 2017. Studies were categorized by phase of the sexual response cycle investigated (wanting, liking, and inhibition) and by methodology (activation vs. connectivity approaches)

\section{Sexual response studies}

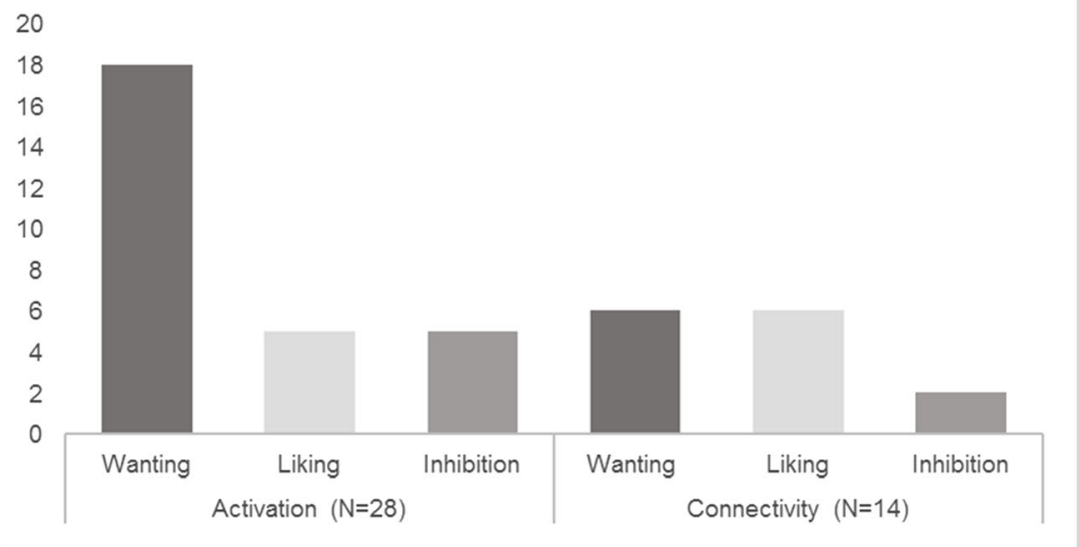

approaches may eliminate unwanted effects of, for instance, adherence to cultural standards on sexual responding.

\section{Wanting Sex: Non-connectivity Approaches}

Neuroscientific interest in the sexual wanting domain is increasingly narrowing down on sexual desire extremes. Several studies using visual sexual stimulation have shown that (perceived) hypersexual behavior (aka compulsive sexual behavior, sexual addiction, or problematic pornography use) is correlated with alterations in neural activation patterns [25-32] and regional brain volume [33•, 34], particularly in areas of the sexual wanting network [14•]. Increased activity to sexual cues has been demonstrated in the VS $[25,27]$ and also in the amygdala in hypersexual men [25, 27, 28], which is suggestive of sexual cue sensitization. This is sometimes taken to support the addiction theory of hypersexuality [35]. Other studies, however, showed negative correlations between sexual cue-induced brain activity and hypersexual symptom severity, suggesting the involvement of different phenomena that are seemingly incompatible with addiction, like response extinction or emotional downregulation [26, 28-30, 34]. These data may not be mutually exclusive. For instance, men with hypersexuality may be both sensitized to sexual cues or contingencies (a feature of addiction) and more easily lose interest or self-regulate if there is no possibility to advance the sexual response (as a learned adaptation). Indeed, in a paradigm with repeated exposure of cues predicting the presentation of a pornographic picture or a monetary reward, cue-induced activity in the ACC decreased faster with repeated exposure in men with hypersexuality-but only for the sexual cues [26].

At the other end of the spectrum, sexual interest/arousal disorder is associated with structural and functional alterations in the sexual wanting network, especially in areas like the ACC, VS, and amygdala, suggesting decreased sexual cue sensitivity [36]. Rupp and colleagues showed that in postpartum women, amygdala responses to emotional pictures (including erotic pictures) was suppressed, indicating decreased sensitivity to emotional salience during the postpartum period [37]. A resting state fMRI study suggested that antidepressant use is associated with altered functional connectivity within the sexual wanting network, especially with regard to the connectivity of the (extended) amygdala. In this study, amygdala connectivity profile prior to antidepressant use reliably predicted if a subject was going to be vulnerable or resilient to antidepressant-related sexual dysfunction [38].

The "sexual wanting network" can be recruited by a range of salient non-erotic stimuli as well [14•], including negative ones [39]. The question then becomes how generic and specific functions work together within this network to produce a distinct sexual interest. Although this question is far from being answered, interesting new insights have been published, mostly on the VS. For instance, VS responses to food and erotic images predicted individual differences in bodyweight and sexual activity, respectively, 6 months later [40]. Another study reported that differences in VS activation for monetary versus erotic cues could be explained by their relative motivational value [41•]. Hence, the VS might signal values for different reward types, but the neural responses for each reward type are unique and are influenced by their salience for a given person. Indeed, relative to healthy controls, men with hypersexuality show stronger VS activity for preferred relative to non-preferred visual erotica [32]. Another area of interest in this context is the OFC, because reward subtypes are processed in different OFC subregions [42]. While primary rewards (like erotic stimuli) activate the OFC posteriorly, secondary rewards (like money) activate a more anterior portion [43]. The OFC is thus a prime candidate to further the study how the brain produces distinct sexual interest and feelings.

Sexual responsiveness shows normal short-term and longterm variability. This has been studied mostly in the context of the sex steroid milieu. Contrary to the biological adage that fertility status drives sexual responsivity, no consistent pattern 
emerges from studies trying to find a relationship between visual stimulation-induced brain activity and menstrual cycle phase [21]. However, Abler and colleagues included an expectancy element in their study and found that, in regularly cycling women, the predicting stimulus (conditioned cue) activated the ACC, OFC, and parahippocampal gyrus more strongly during the luteal phase than the follicular phase. Activation in these areas was stronger in regularly cycling women, as compared to those on oral contraceptives [44].

Testosterone is seen as the gonadal hormone most pertinent to human sexual responsiveness [45, 46]. Indeed, brains of genetic men without androgen function (complete androgen insensitivity syndrome, "46XY women") responded in a typical female-like fashion to visual erotic stimulation, that is, similar to male controls but at weaker strength [47]. Because in both 46XY and genetic women, there is less central testosterone function than in men; it was concluded that testosterone rather than genetic sex determines brain activity patterns during sexual stimulation. Yet, a DTI experiment studying brain structure in transgender and cisgender women and men found white matter variation that could not be accounted for by differences in testosterone function. Trans people exhibited white matter values midway between male and female cisgender controls, despite gonadal hormone levels being either typically male or female (depending on whether they were transgender women or transgender men) [48].

\section{Wanting Sex: Connectivity Approaches}

Functional connectivity within the sexual wanting network has recently been investigated using the PPI approach, mainly in the context of (perceived) hypersexuality. Men with hypersexuality and controls both show increased functional connectivity of the ACC with both the right VS and right amygdala when viewing erotica, but the strongest positive correlation with reported sexual desire was found for ACC-subcortical connectivity in hypersexuality [25]. After many repetitions of sexual stimulation, functional connectivity of the ACC with the right VS and with the bilateral hippocampus was stronger in men with hypersexuality than in controls. Intriguingly, this increased functional connectivity within the sexual wanting network occurred in the presence of decreased ACC activity [26]. This could signify a habituation effect, but more research is required to explore this phenomenon. Another study used a design with cues predicting pornographic or non-erotic stimuli and found decreased functional connectivity between the VS and ventromedial PFC for men with hypersexuality compared to controls [28]. Since altered VS-prefrontal coupling has been associated with impulsivity control, substance abuse, and pathological gambling [49-51], these findings could be an indication of inhibition impairment in men with hypersexuality. Two other studies employed a resting state design, showing that $(i)$ reported hours of watching pornography (per week) are negatively correlated with resting state connectivity between the right caudate nucleus and left dorsolateral PFC and (ii) subjects diagnosed with compulsive sexual behavior have decreased functional connectivity between the left amygdala and bilateral dorsolateral PFC $[33 \bullet, 34]$. These studies indicate that increases in sexual behavior are marked by altered prefrontal control mechanisms. Together, these connectivity studies strengthen the assumption that the "sexual wanting" pattern identified by activation studies is indeed the resemblance of a true functional network, because a subset of its constituent brain areas alters their communication when sexual incentives are presented, while the strength of this interaction reflects the sexual behavioral phenotype. Fronto-striatal connectivity and VS connectivity hold high promise as research avenues into the fundamentals of (aberrant) sexual wanting.

\section{Liking Sex}

Brain imaging paradigms employing stronger and more prolonged visual sexual stimulation (for example, porn movies), or tactile genital stimulation, are likely to model (elements of) having sex (e.g., evoke physiological genital responses and sexual liking). As indicated earlier, this phase recruits a brain network that is relatively distinct from that recruited during wanting sex, and this is especially so in men [3••, 13, 14•, 20]. Liking sex has also seen more studies focusing on brain connectivity than wanting sex has (Fig. 1).

One disorder that is currently receiving particular attention is psychogenic erectile dysfunction ( $p E D)$. This condition has been associated with increased or decreased gray matter volume in many brain areas, including those belonging to sexual wanting and liking networks [52, 53•]. It has also been associated with persistent sexual wanting network activation (superior parietal lobule specifically), possibly resulting in a failure to shift to the next phase of the sexual response cycle [54]. Interestingly, $\mathrm{pED}$ is now predominantly being studied with structural or resting state neuroimaging research paradigms, contrary to other sexual disorders that are dominated by taskbased paradigms. Altered functional connectivity within and beyond sexual wanting and liking networks has been identified. For instance, the right lateral OFC was found to have aberrant structural connectivity with areas in the parietal lobe in $\mathrm{pED}$ [53•]. In a resting state fMRI study, pED subjects showed altered functional connectivity of the right anterior insula (an area integral to interoception and emotion regulation) with the dorsolateral PFC and right parietotemporal junction, compared to controls [55]. This indicates that $\mathrm{pED}$ may come with an abnormal representation of bodily states (including erection) and/or excessive inhibition control. Interestingly, when subjects viewed a porn movie for the duration of the experiment (instead of resting), reduced functional connectivity of the right insula was also found in individuals with pED relative to healthy volunteers [56]. Even though the 
experimental paradigms differ, the results seem congruent, again involving components of both wanting and liking networks that also show structural degradation in pED [53•].

None of the studies discussed so far have considered wholebrain connectivity. In fact, the first study to do this was published only 2 years ago. Zhao and colleagues applied graph analysis methods to structural data to study diverging brain connectivity profiles in pED subjects [57••]. As expected, the whole-brain connectivity profile of $\mathrm{pED}$ subjects and healthy subjects had a small-world organization characterized by both networks for local specialization and global integration. However, in pED, the balance was shifted towards local specialization, possibly resulting in poorer integration of network activity. Indeed, fewer hubs (integrating areas) were identified in $\mathrm{pED}$ than in controls, indicating overall poorer global integration.

Genital stimulation is the primary source of sexual pleasure (liking) in the brain and is a key contributor to sexual arousal [13]. Yet, very little is known about the brain's role in sexual development of genital sensations. Some new insights are provided by research in spina bifida patients who underwent a surgical reinnervation of their lifelong insensate penis to improve their sexual function. Stimulation of the glans penis (reinnervated by a groin nerve) and the intact groin area (contralateral to the area that provided the donor nerve) activated the same area of the primary somatosensory cortex, as expected. However, primary somatosensory cortex was functionally connected with the MCC and operculum-insular cortex during penis stimulation, but not during groin stimulation [58]. Wise et al. studied to what extent brain activation overlaps or differs for both physical and imagined genital stimulation in women [59]. One of the more interesting results is that imagined dildo stimulation activated hippocampus/amygdala, insula, VS, ventromedial PFC, and somatosensory cortices more than imagined speculum stimulation. Another recent study in masochists showed decreased functional connectivity of the parietal operculum with the bilateral insulae and operculum when they received painful stimuli in masochistic context, indicating a network for pain modulation in favor of sexual arousal [60]. Even when candidate areas have been suggested, clearly more work is needed to identify the key areas that govern not only the sexual interpretation of genital sensation in relation to context, but also the transition of genital to sexual sensations in normal sexual development.

\section{Inhibiting Sex}

From a behavioral point of view, the potential to inhibit or control a sexual response is equally critical as being able to respond sexually. Thus, in the brain, there must be a continuous interplay between systems that promote approach and systems that promote avoidance. A more or less consistent finding is that prefrontal areas tend to show exaggerated activity in subjects with hyposexual behavior [61-63]. However, breast cancer survivors who report distress about their loss of sexual desire showed reduced activity in the dorsolateral PFC and ACC when viewing pornographic pictures, compared to non-distressed breast cancer survivors [64]. This result seems counterintuitive, but chronic stressors are associated with prefrontal hyporegulation of subcortical areas [65]. Clinical findings confirm that prefrontal function needs to be within an optimal range for sex to function normally [66], illustrating the very important point that normal brain function requires optimal balancing of brain systems.

Victor and colleagues performed an interesting fMRI study focusing on the VS-amygdala balance as an index of the individual trait to inhibit sexual responding [67••]. Their hypothesis was that VS responding to appropriate sexual stimuli is only half of the story; in order for a sexual response to advance, the amygdala should also deactivate to "release the brake." This is in line with studies showing decreased medial temporal lobe activity during high sexual arousal (e.g., see [14•]). Interestingly, high VS and low amygdala activity during a non-erotic impulsivity test was indeed found to predict a higher number of sex partners 6 months after the study, but in male participants only; in women, the highest number of new sex partners was predicted by a combination of high VS and amygdala activity [67••]. Importantly, VS and amygdala activity might also reflect a specific negative appreciation of sexual stimulation. In a recent fMRI study which included an implicit association test, women viewed images of explicit penetrative sex. Contrary to what might be expected, VS activity (and the basal forebrain-amygdala continuum) did not reflect approach or positive interest; instead, those subjects that showed the strongest automatic avoidance of extreme porn had the strongest porn-induced VS response [68•]. Together, these findings clearly demonstrate that detecting a salient sexual stimulus is not sufficient to advance a sexual response, but rather, that sexual response results from a complex interplay between approach and avoidance, the neural mechanisms of which are only beginning to be unveiled.

\section{Conclusion and Future Directions}

Human sexuality does not rely on a single "sex nucleus." Rather, it involves many-sometimes quite generic - brain functions including those for arousal, reward, memory, cognition, self-referential thinking, and social behavior. As clearly

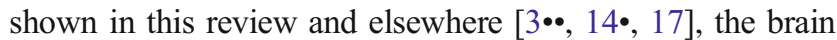
areas that have been associated with human sexuality are spatially remote. From this point of view, studying the connectivity of the brain is much more intuitive than studying separate "activations," and in fact, studying the nature of the connectivity between brain regions has been a common practice in animal models of human sexual behavior for many decades already (see e.g., [46]). Every fraction of a second, billions of 
neurons "talk" to each other by virtue of an unthinkable wiring creating even more complex neural networks. It is by understanding how these networks operate - alone, but preferably in conjunction with each other - that we can begin to understand the neural mechanisms that critically regulate human sexual function and that can account for non-organic sexual dysfunction. Currently, the urgency to take such an approach seems more pertinent in other fields of sexuality research, like gender identity/transsexuality and child sexual offending. For instance, a recent study used structural MRI data to define regions with gray matter deficits in pedophilia and then assessed a reliable functional connectivity profile of these areas using a large brain database (data from 7500 brain experiments were used). It turned out that morphologically altered areas in pedophilia are functionally connected primarily with areas important for sexual responsiveness, i.e., areas of the sexual wanting and liking networks [69••]. This is strongly suggestive of a situation where a functional sexual response is connected to - or controlled by - brain regions with significant morphological deficits. As another example of more sophisticated application of neuroimaging to the study of human sexuality, a recent study used graph analysis to show that, relative to cisgenders, transgender people have a stronger local specialization of their somatosensory network, characterized by more and stronger local connections [70]. Most likely, this underlies their differential body perception. By approaching the brain as a connected organ, studies such as these capture the essence of brain function much more accurately, increasing the likelihood of finding useful biomarkers and targets for intervention. We strongly encourage that such methods be used more to study the human sexual response, because accepting that conditions like sexual pain/penetration disorder, sexual interest /arousal disorder, hypersexual complaints, premature ejaculation, persistent genital arousal disorder, and anorgasmia originate in the brain is not enough; sexual dysfunctions are complex, multidimensional, and multifactorial and by their very nature, suitable to be studied from a "connectivity" perspective.

\section{Compliance with Ethical Standards}

Conflict of Interest The authors declare that they have no conflict of interest.

Human and Animal Rights and Informed Consent All reported studies/experiments with human or animal subjects performed by the authors have been previously published and complied with all applicable ethical standards (including the Helsinki declaration and its amendments, institutional/national research committee standards, and international/national/institutional guidelines).

Open Access This article is distributed under the terms of the Creative Commons Attribution 4.0 International License (http:// creativecommons.org/licenses/by/4.0/), which permits unrestricted use, distribution, and reproduction in any medium, provided you give appropriate credit to the original author(s) and the source, provide a link to the Creative Commons license, and indicate if changes were made.

\section{References}

Papers of particular interest, published recently, have been highlighted as:

- Of importance

• Of major importance

1. Joel D, Berman Z, Tavor I, et al. Sex beyond the genitalia: the human brain mosaic. Proc Natl Acad Sci. 2015;112:15468-73 Elaborate quantitative meta-analysis (including connectivity) showing that many people do not have a 'male' or a 'female' brain.

2. Eickhoff SB, Laird AR, Grefkes C, Wang LE, Zilles K, Fox PT. Coordinate-based activation likelihood estimation meta-analysis of neuroimaging data: a random-effects approach based on empirical estimates of spatial uncertainty. Hum Brain Mapp. 2009;30:290726.

3.• Poeppl TB, Langguth B, Laird AR, Eickhoff SB. The functional neuroanatomy of male psychosexual and physiosexual arousal: a quantitative meta-analysis. Hum Brain Mapp. 2014;35:1404-21. Example of a systematic and quantitative approach to establish patterns of brain areas that are involved of different sexual response cycle phases.

4. O'Reilly JX, Woolrich MW, Behrens TEJ, Smith SM, JohansenBerg H. Tools of the trade: psychophysiological interactions and functional connectivity. Soc Cogn Affect Neurosci. 2012;7:604-9.

5. Hyvärinen A. Fast and robust fixed-point algorithms for independent component analysis. IEEE Trans Neural Netw. 1999;10:62634.

$6 . \bullet$ van den Heuvel MP, Hulshoff Pol HE. Exploring the brain network: a review on resting-state fMRI functional connectivity. Eur Neuropsychopharmacol. 2010;20:519-34. Accessible resource for further information on functional brain networks.

7. Hjelmervik H, Hausmann M, Osnes B, Westerhausen R, Specht K. Resting states are resting traits - an fMRI study of sex differences and menstrual cycle effects in resting state cognitive control networks. PLoS One. 2014;9:32-6.

8. Friston K, Moran R, Seth AK. Analysing connectivity with Granger causality and dynamic causal modelling. Curr Opin Neurobiol. 2013;23:172-8.

9.• Sporns O. Structure and function of complex brain networks. Dialogues Clin Neurosci. 2013;15:247-62. An accessible introduction to methodological approaches for the study of complex brain connectivity.

10. Bullmore ET, Sporns O. Complex brain networks: graph theoretical analysis of structural and functional systems. Nat Rev Neurosci. 2009;10:186-98.

11. He Y, Chen ZJ, Evans AC. Small-world anatomical networks in the human brain revealed by cortical thickness from MRI. Cereb Cortex. 2007;17:2407-19.

12. Masters WH, Johnson VE. Human sexual response. Hum Sex Response. 1966. https://doi.org/10.1016/B978-0-444-63247-0. 00002-X.

13. Georgiadis JR, Kringelbach ML, Pfaus JG. Sex for fun: a synthesis of human and animal neurobiology. Nat Rev Urol. 2012;9:486-98.

14. Georgiadis JR, Kringelbach ML. The human sexual response cycle: brain imaging evidence linking sex to other pleasures. Prog Neurobiol. 2012;98:49-81. Meta-analysis emphasizing the similarity of sex to other pleasures, and proposing the Human Sexual Pleasure Cycle as a model to study sexual responses.

15. Robinson TE, Berridge KC. The neural basis of drug craving: an incentive-sensitization theory of addiction. Brain Res Rev. 1993;18: 247-91. 
16. Toates FM. Motivational systems. Curr Opin Neurobiol. 1986;20: 188.

17. Stoléru S, Fonteille V, Cornélis C, Joyal C, Moulier V. Functional neuroimaging studies of sexual arousal and orgasm in healthy men and women: a review and meta-analysis. Neurosci Biobehav Rev. 2012;36:1481-509.

18. Ponseti J, Granert O, van Eimeren T, Jansen O, Wolff S, Beier K, et al. Human face processing is tuned to sexual age preferences. Biol Lett. 2014;10:20140200.

19. Poeppl TB, Langguth B, Rupprecht R, Laird AR, Eickhoff SB. A neural circuit encoding sexual preference in humans. Neurosci Biobehav Rev. 2016;68:530-6.

20. Poeppl TB, Langguth B, Rupprecht R, Safron A, Bzdok D, Laird AR, et al. The neural basis of sex differences in sexual behavior: a quantitative meta-analysis. Front Neuroendocrinol. 2016;43:28-43.

21. Levin RJ, Both S, Georgiadis J, Kukkonen T, Park K, Yang CC. The physiology of female sexual function and the pathophysiology of female sexual dysfunction (Committee 13A). J Sex Med. 2016;13:733-59.

22. Wehrum-Osinsky S, Klucken T, Kagerer S, Walter B, Hermann A, Stark R. At the second glance: stability of neural responses toward visual sexual stimuli. J Sex Med. 2014;11:2720-37.

23. Wernicke M, Hofter C, Jordan K, Fromberger P, Dechent P, Müller JL. Neural correlates of subliminally presented visual sexual stimuli. Conscious Cogn. 2017;49:35-52.

24. Jordan K, Wieser K, Methfessel I, Fromberger P, Dechent P, Müller JL. Sex attracts - neural correlates of sexual preference under cognitive demand. Brain Imaging Behav. 2017; 1-18.

25. Voon V, Mole TB, Banca P, et al. Neural correlates of sexual cue reactivity in individuals with and without compulsive sexual behaviours. PLoS One. 2014. https://doi.org/10.1371/journal.pone. 0102419.

26. Banca P, Morris LS, Mitchell S, Harrison NA, Potenza MN, Voon V. Novelty, conditioning and attentional bias to sexual rewards. J Psychiatr Res. 2016;72:91-101.

27. Politis M, Loane C, Wu K, O'Sullivan SS, Woodhead Z, Kiferle L, et al. Neural response to visual sexual cues in dopamine treatmentlinked hypersexuality in Parkinson's disease. Brain. 2013;136:400-11.

28. Klucken T, Wehrum-Osinsky S, Schweckendiek J, Kruse O, Stark R. Altered appetitive conditioning and neural connectivity in subjects with compulsive sexual behavior. J Sex Med. 2016;13:627-36.

29. Steele VR, Staley C, Fong T, Prause N. Sexual desire, not hypersexuality, is related to neurophysiological responses elicited by sexual images. Socioaffect Neurosci Psychol. 2013;3:20770.

30. Prause N, Steele VR, Staley C, Sabatinelli D, Hajcak G. Modulation of late positive potentials by sexual images in problem users and controls inconsistent with "porn addiction". Biol Psychol. 2015;109:192-9.

31. Seok J-W, Sohn J-H. Neural substrates of sexual desire in individuals with problematic hypersexual behavior. Front Behav Neurosci. 2015;9:1-11.

32. Brand M, Snagowski J, Laier C, Maderwald S. Ventral striatum activity when watching preferred pornographic pictures is correlated with symptoms of Internet pornography addiction. NeuroImage. 2016;129:224-32.

33. Schmidt C, Morris LS, Kvamme TL, Hall P, Birchard T, Voon V. Compulsive sexual behavior: prefrontal and limbic volume and interactions. Hum Brain Mapp. 2017;38:1182-90. Example of a study using resting-state data to demonstrate changes in hypersexual compared to sexually asymptomatic volunteers at the functional network level.

34. Kühn S, Gallinat J. Brain structure and functional connectivity associated with pornography consumption. JAMA Psychiatry. 2014;71:827.
35. Potenza MN, Gola M, Voon V, Kor A, Kraus SW. Is excessive sexual behaviour an addictive disorder? Lancet Psychiatry. 2017;4:663-4.

36. Bloemers J, Scholte HS, van Rooij K, Goldstein I, Gerritsen J, Olivier B, et al. Reduced gray matter volume and increased white matter fractional anisotropy in women with hypoactive sexual desire disorder. J Sex Med. 2014;11:753-67.

37. Rupp HA, James TW, Ketterson ED, Sengelaub DR, Ditzen B, Heiman JR. Lower sexual interest in postpartum women: relationship to amygdala activation and intranasal oxytocin. Horm Behav. 2013;63:114-21.

38. Metzger CD, Walter M, Graf H, Abler B. SSRI-related modulation of sexual functioning is predicted by pre-treatment resting state functional connectivity in healthy men. Arch Sex Behav. 2013;42: 935-47.

39. Borg C, Georgiadis JR, Renken RJ, Spoelstra SK, Schultz WW, De Jong PJ. Brain processing of visual stimuli representing sexual penetration versus core and animal-reminder disgust in women with lifelong vaginismus. PLoS One. 2014. https://doi.org/10.1371/ journal.pone.0084882.

40. Demos KE, Heatherton TF, Kelley WM. Individual differences in nucleus accumbens activity to food and sexual images predict weight gain and sexual behavior. J Neurosci. 2012;32:5549-52.

41. Sescousse G, Li Y, Dreher JC. A common currency for the computation of motivational values in the human striatum. Soc Cogn Affect Neurosci. 2015;10:467-73. Study demonstrating the important fact that recruitment of the wanting network is not specific to sex.

42. Sescousse G, Redoute J, Dreher J-C. The architecture of reward value coding in the human orbitofrontal cortex. J Neurosci. 2010;30:13095-104.

43. Li Y, Sescousse G, Amiez C, Dreher J-C. Local morphology predicts functional organization of experienced value signals in the human orbitofrontal cortex. J Neurosci. 2015;35:1648-58.

44. Abler B, Kumpfmüller D, Grön G, Walter M, Stingl J, Seeringer A. Neural correlates of erotic stimulation under different levels of female sexual hormones. PLoS One. 2013. https://doi.org/10.1371/ journal.pone.0054447.

45. Agmo A. Functional and dysfunctional sexual behavior: a synthesis of neuroscience and comparative psychology. San Diego: Academic Press; 2011.

46. Pfaus JG. Pathways of sexual desire. J Sex Med. 2009;6:1506-33.

47. Hamann S, Stevens J, Vick JH, Bryk K, Quigley CA, Berenbaum SA, et al. Brain responses to sexual images in 46,XY women with complete androgen insensitivity syndrome are female-typical. Horm Behav. 2014;66:724-30.

48. Kranz GS, Hahn A, Kaufmann U, et al. White matter microstructure in transsexuals and controls investigated by diffusion tensor imaging. J Neurosci. 2014;34:15466-75.

49. Diekhof EK, Gruber O. When desire collides with reason: functional interactions between anteroventral prefrontal cortex and nucleus accumbens underlie the human ability to resist impulsive desires. $\mathrm{J}$ Neurosci. 2010;30:1488-93.

50. Motzkin JC, Baskin-Sommers A, Newman JP, Kiehl KA, Koenigs M. Neural correlates of substance abuse: reduced functional connectivity between areas underlying reward and cognitive control. Hum Brain Mapp. 2014;35:4282-92.

51. Cilia R, Cho SS, van Eimeren T, Marotta G, Siri C, Ko JH, et al. Pathological gambling in patients with Parkinson's disease is associated with fronto-striatal disconnection: a path modeling analysis. Mov Disord. 2011;26:225-33.

52. Cera N, Delli Pizzi S, Di Pierro ED, Gambi F, Tartaro A, Vicentini $\mathrm{C}$, et al. Macrostructural alterations of subcortical grey matter in psychogenic erectile dysfunction. PLoS One. 2012;7:e39118.

53. Zhao L, Guan M, Zhang X, et al. Structural insights into aberrant cortical morphometry and network organization in psychogenic 
erectile dysfunction. Hum Brain Mapp. 2015;36:4469-82. Innovative experimental design which uses cortical thickness measures obtained from structural MRI to explore structural connectivity changes in $\mathbf{p E D}$.

54. Cera N, di Pierro ED, Sepede G, et al. The role of left superior parietal lobe in male sexual behavior: dynamics of distinct components revealed by fMRI. J Sex Med. 2012;9:1602-12.

55. Wang Y, Dong M, Guan M, Wu J, He Z, Zou Z, et al. Aberrant insula-centered functional connectivity in psychogenic erectile dysfunction patients: a resting-state fMRI study. Front Hum Neurosci. 2017;11:221.

56. Cera N, Di Pierro ED, Ferretti A, Tartaro A, Romani GL, Perrucci MG. Brain networks during free viewing of complex erotic movie: new insights on psychogenic erectile dysfunction. PLoS One. 2014. https://doi.org/10.1371/journal.pone.0105336.

57.• Zhao L, Guan M, Zhu X, et al. Aberrant topological patterns of structural cortical networks in psychogenic erectile dysfunction. Front Hum Neurosci. 2015;9:1-16. The first neuroimaging study to use whole brain connectivity measures in relation to sexual function.

58. Kortekaas R, Nanetti L, Overgoor MLE, de Jong BM, Georgiadis JR. Central somatosensory networks respond to a de novo innervated penis: a proof of concept in three spina bifida patients. J Sex Med. 2015;12:1865-77.

59. Wise NJ, Frangos E, Komisaruk BR. Activation of sensory cortex by imagined genital stimulation: an fMRI analysis. Socioaffect Neurosci Psychol. 2016;6:31481.

60. Kamping S, Andoh J, Bomba IC, Diers M, Diesch E, Flor H. Contextual modulation of pain in masochists. Pain. 2016;157: 445-55.

61. Stoléru S, Redouté J, Costes N, Lavenne F, Le Bars D, Dechaud H, et al. Brain processing of visual sexual stimuli in men with hypoactive sexual desire disorder. Psychiatry ResNeuroimaging. 2003;124:67-86.

62. Bianchi-Demicheli F, Cojan Y, Waber L, Recordon N, Vuilleumier $\mathrm{P}$, Ortigue $\mathrm{S}$. Neural bases of hypoactive sexual desire disorder in women: an event-related fMRI study. J Sex Med. 2011;8:2546-59.
63. Arnow BA, Millheiser L, Garrett A, et al. Women with hypoactive sexual desire disorder compared to normal females: a functional magnetic resonance imaging study. Neuroscience. 2009;158:484-502.

64. Versace F, Engelmann JM, Jackson EF, Slapin A, Cortese KM, Bevers TB, et al. Brain responses to erotic and other emotional stimuli in breast cancer survivors with and without distress about low sexual desire: a preliminary fMRI study. Brain Imaging Behav. 2013;7:533-42.

65. Gagnepain P, Hulbert J, Anderson MC. Parallel regulation of memory and emotion supports the suppression of intrusive memories. $\mathrm{J}$ Neurosci. 2017;37:6423-41.

66. Rees PM, Fowler CJ, Maas CP. Sexual function in men and women with neurological disorders. Lancet. 2007;369:512-25.

67.• Victor EC, Sansosti AA, Bowman HC, Hariri AR. Differential patterns of amygdala and ventral striatum activation predict genderspecific changes in sexual risk behavior. J Neurosci. 2015;35:8896900. Example of an approach where information about nonsexual brain function can be predictive of sexual behavior.

68. Borg C, de Jong PJ, Georgiadis JR. Subcortical BOLD responses during visual sexual stimulation vary as a function of implicit porn associations in women. Soc Cogn Affect Neurosci. 2014;9:158-66. Study demonstration that increased activity in sexual wanting areas does not necessarily reflect a positive attitude towards sexual stimuli.

69.• Poeppl TB, Eickhoff SB, Fox PT, Laird AR, Rupprecht R, Langguth $\mathrm{B}$, et al. Connectivity and functional profiling of abnormal brain structures in pedophilia. Hum Brain Mapp. 2015;36: 2374-86. A mixture of meta-analysis, connectivity, and structural data. Shows that regions with altered morphology in pedophilia are functionally connected to areas of sexual response brain networks.

70. Lin CS, Ku HL, Chao HT, Tu PC, Li CT, Cheng CM, Su TP, Lee YC, Hsieh JC. Neural network of body representation differs between transsexuals and cissexuals. PLoS One. 2014. https://doi.org/ 10.1371/journal.pone.0085914. 